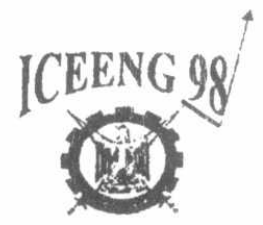

\title{
THE EFFECT OF OVERLAPPING PULSES ON THE OPERATION OF AN AU'TOMATIC ESM RECEIVING SYSTEM
}

\author{
${ }^{*}$ Khairy El-Barbary, and "Hosam E. Abou-bakr
}

\begin{abstract}
Radar electronic support measures (ESM) systems perform the functions of threat detection and area surveillance to determine the identity and bearing of surrounding radar emitters. These ESM receivers have a wide frec $q$ uency band and their antennas have a wide coverage pattern. These features of the ESM receiver are necessary to increase the instantaneous coverage and to enhance the probability of in'cerception. However, the ESM receiver is vulnerable to overlapping pulses, these pulses which arrive simultaneously or partially are time overlapped to the ESM receiver input In this work a cueuing model is performed to describe the arrival process of radar pulses at the input of an ESM receiver. From this arrival model, the probability of pulse overlapping is determined for an arbitrary arrival rate. The effect of this undesired pulse overlapping on the measurernents performed by an ESM receiver and consequently the output digital words that describe the arrival pulse train is evaluated in two cases. The first case which is denoted as parajyzable receiver type, where the E.SM receiver can only process a pulse which arrives after a fixed time from the last pulse processed. The second case is denoted as non paralyzable receiver, where the receiver is ready to process a new nulse as soon as the life time of the previous pulse is expired. A comruter program is constructed to simulate the operation of an ESM receiver in these two cases. The results show that the ratio of successfully processed pulses in case of non paralyzable processing is larger than the case of a paralyzable processing .
\end{abstract}

\section{INT'RODUCT'YON}

T.jue to the dense environments surroundi..g an ESM 1 receiver, it is expected that the antenna of an ESM receiver will be simultaneously illımincited by a relatively large number of periodic radars. Because of this dense environments the received pulses from different radars will emerge together; and sometimes they will be overlapped, which affects the bəhavior of the ESM receiver. In section 2 a mathematical model representing the arrival process is constructed. The probability density function of the interarrival time between successive pulses and the average arrival rate are evaluated. In sections 3 and 4 we will discuss the concept of operation of an ESM receiver and deduce the probability density function of the times between the output processed pulses from the receiver. In section 5 we drive an analytic expression for calculation of the rate of output processed pulses from the receiver. The ratio of successfully processed pulses in both cases of paralayzable and non- paralayzable receiver models is evaluated in terms of the input arrival rate and the processing speed. Simulation results are presented in number of figures which indicate the

Electronic and Electrical Engineering Department, Military Technical College, Cairo, Egypt 
Proceeding of the $1^{\text {st }}$ ICEENG conference, 24-26 March, 1998.

$R G .3 \quad 392$

difference between the paralayzable and non -paralayzable processing of an intercepted radar pulses .

\section{MATHEMATICAL MODEL FOR THE ARRIVAL PROCESS TO AN ESM RECEIVER}

There are many publications which concern the characterization of the arrival process to the input of an ESM receiver. It is proved that, [1] if $\mathrm{N}$-radars with rotating antennas illuminate an ESM receiver, the average arrival rate at the input of the ESM receiver is approximately equal to the sum of the pulse repetition frequencies of the existing radars in the environments. This relation is represented as :

$$
\lambda=\sum_{i=1}^{N} P R F_{i}
$$

where,

$\lambda$ is the average pulse rate at the input of an ESM receiver

$\mathrm{PRF}_{\mathrm{i}}$ is the pulse repetition frequency of the $i^{\text {th }}$ radar

Moreover, the number of received pulses during an arbitrary time interval $t$ follows the Poisson distribution, [1] with an average rate $\lambda$ pulses per second thus

$$
\mathrm{P}_{\mathrm{n}}(\mathrm{t})=\frac{(\lambda t)^{n}}{n !} e^{-\lambda t}
$$

where,

$P_{n}(t)$ is the probability that $n$ pulses arrive in $t$ seconds at the input of an ESM receiver

It is easy to prove that the interarrival time between successive received pulses is a random variable which follows the negative exponential distribution, [2] that;

$$
\mathrm{f}_{\mathrm{T}}(\mathrm{t})=\lambda \mathrm{e}^{-\lambda \mathrm{t}}
$$

Where :

$\mathrm{T}$

is the interarrival time betwe-in received pulses

$\mathrm{f}_{\mathrm{T}}(\mathrm{t})$ is the probability density function of $\mathrm{T}$.

A computer program is constructed to simulate the environments of an ESM receiver and verify the obtained analytic formulation. Fig. 1 shows the relationship between the number of radars in the environments of an ESM receiver and average input arrival rate. The solid curve is calculated using equation (1), whereas the dashed line is evaluated using computer simulation, we can see that the two curves are approximately identical. 
Proceeding of the $1^{\text {st }}$ ICEENG conference, 24-26 March, 1998

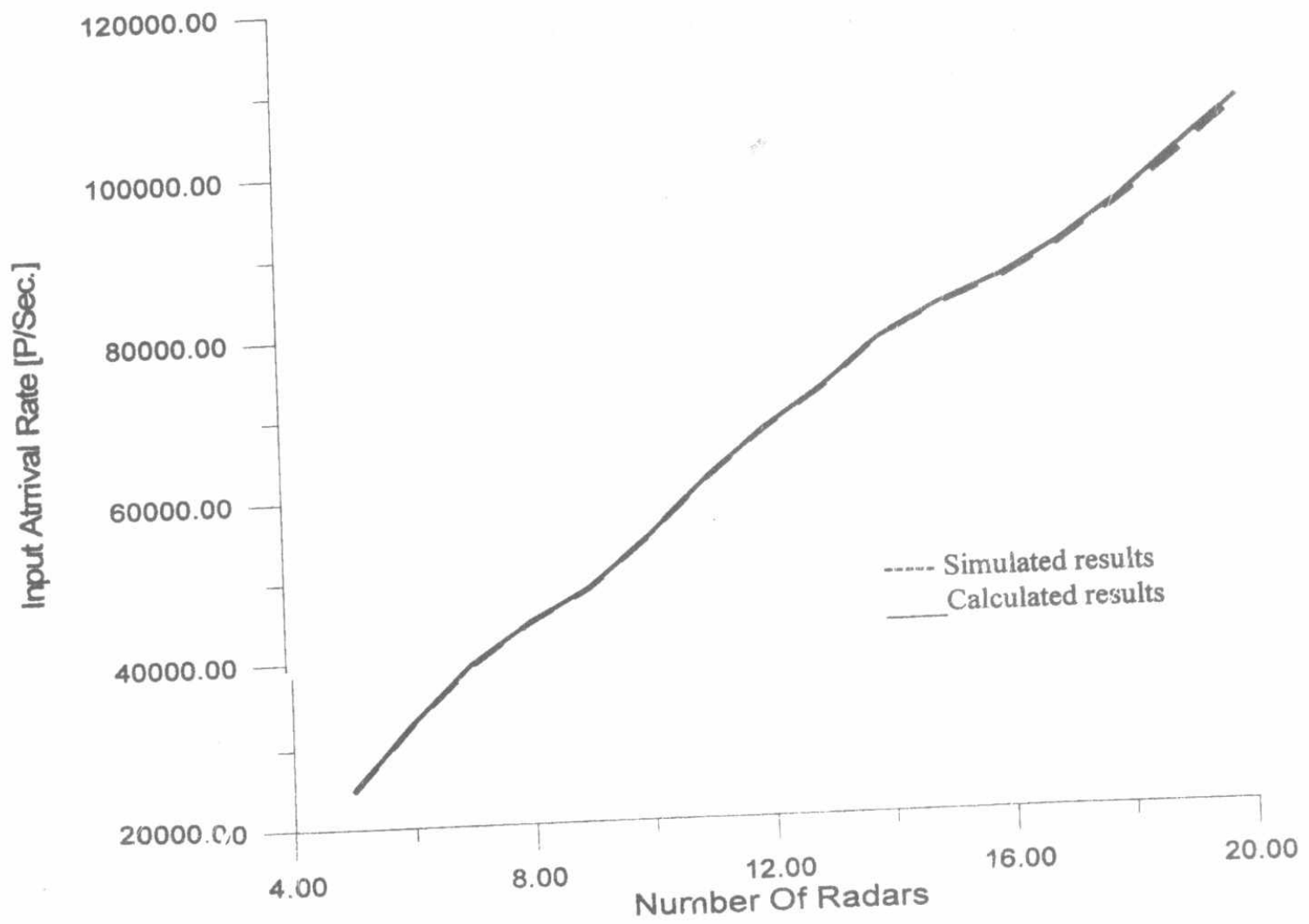

Fig. 1. The input arrival rate as function of the number of radars illuminating the ESM receiver

\section{RECEIVING MODEL}

An intercepted pulse is processed by an ESM receiver where different parameters of this pulse are measured. These pulse parameters are encoded in the form of digital word called pulse descriptive vector (PDV).Commonly the PDV has the formats as shown in Fig. 2.

\begin{tabular}{|l|l|l|l|l|}
\hline $\mathrm{AC}, \mathrm{A}\left[\mathrm{M}_{1}\right.$ bits $]$ & $\mathrm{RF}\left[\mathrm{M}_{2}\right.$ bits $]$ & PW $\left[\mathrm{M}_{3}\right.$ bits $]$ & TOA $\left[\mathrm{M}_{4}\right.$ bits $]$ & PA $\left[\mathrm{M}_{5}\right.$ bits $]$ \\
\hline
\end{tabular}

Fig. 2. Representation of the information field of a PDV

Where,

$\mathrm{M}_{1}$

$\mathrm{M}_{2}$

$\mathrm{M}_{3}$

$\mathrm{M}_{4}$

is the number of bits representing the measured angle of arrival (AOA) of the intercepted pulse. is the number of bits representing the measured radio frequency (RF) of the intercepted pulse. is the number of bits representing the measured pulse width (PW) of the intercepted pulse, is the number of bits representing the measured time of arrival (TOA) of the intercepted pulse. 
$\mathrm{M}_{5} \quad$ is the number of bits representing the measured pulse amplitude (PA) of the intercepted pulse.

The length $\mathrm{L}$ of the PDV is calculated according to the relationship

$$
\mathrm{L}=\sum_{i=1}^{5} M r_{i}
$$

The length of each sub field $M_{i}$ depends on the required resolution and accuracy of measurements, while as the total length $\mathrm{L}$ of the PI)V determines the required speed of the deinterleaver processor .The service time of an intercepted pulse namely the time required by the receiver and the encoder to produce a PDV depends on the concept of operation of the ESM receiver. Two concepts of operations are generally used in an ESM receiver. The first concept of operation is; the paralalayzable receiver mode in which the receiver can only process pulse which arrives $\tau$ seconds after the previous received pulse. The value of the fixed service time $\tau$ is approximately equal to the maximum pulse width expected to be intercepted by the ESM receiver. The second concept of operation is the non paralayzable receiver mode in which as soon as the duration of the previous received pulse has been expired the receiver will be ready to process a new pulse. From the above two modes of operations it is clear that the distinction between the first and second concept of operation is that the first extends the blocking period till the end of the fixed service time , while the second only during the life time of the pulse.

The process of detecting a pulse, measuring its parameters and encoding it into PDV is considered as the service provided by an ESM receiver to an intercepted pulse. Thus the process could be modeled as a queuing problem whose elements are :

i-The arrival process to the input of the ESM receiver, which is represented as Poisson points, with average arrival rate $\lambda$.

ii- The server which consists of the ESM receiver and the encoder.

iii-The service time for each pulse which equals to the time required to measure its parameters and encode it into a PDV.

This queuing model, that describes the receiving operation can be represented as shown in Fig.3.

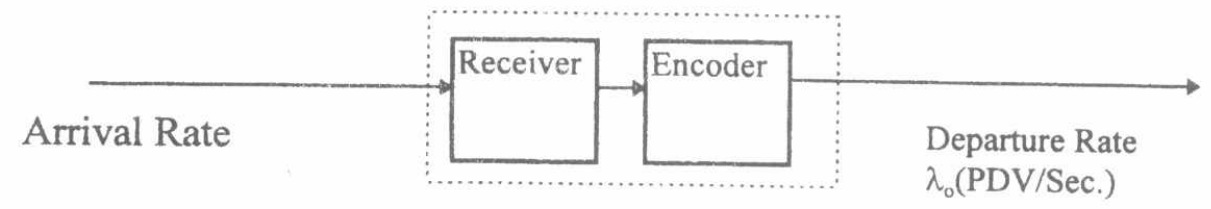

Fig. 3. Queueing model of the receiver and encoder subsystem

The output process has an average departure rate $\lambda_{0}$, which differs from the input arrival rate $\lambda$ due to the concept of operations of the receiver and the time overlapping problem of the received pulses.

\section{DESCRIBTION OF THE DEPARTURE PROCESS}

Suppose that $\mathrm{n}$ pulses arrive at the input of the ESM receiver during $\mathrm{t}$ seconds. If $\mathrm{k}$ pulses of them will be successfully processed by the receiver and emerge from the receiver subsystem in the form of $k$ PDVs, then $n-k$ pulses will not be processed or missed. Assuming that $P_{o}$ represents the 
probability that a single pulse will be successfully processed, then the number of successfully processed pulses during the time interval $t$ follows the binomial distribution that

$$
\mathrm{P}_{\mathrm{k} / \mathrm{n}}(\mathrm{t})=\left({ }_{k}^{n}\right) \mathrm{P}_{0}{ }^{\mathrm{k}}(1-\mathrm{Po})^{n-k}
$$

where,

$\mathrm{P}_{k / n}(t)$

is the probability that $\mathrm{k}$ PDVs will emerge from the receiver/encoder subsystem given that $\mathrm{n}$ pulses are received at its input during time $\mathrm{t}$.

1-Po is the probability that a pulse will not be processed successfully due to the blocking of the receiver by a previous pulse

Remember that the number of received pulses at the ESM receiver input is random variable, and distributed according to Poisson distribution with parameter $\lambda$

So, we can drive a mathematical formula for $P_{k}(t)$ which is the probability that $k$ PDVs will emerge from the receiver in $t$ second whatever the number of receiving pulses at the input of the ESM receiving antenna. Applying the low of total probability we get

$$
\begin{aligned}
& \mathrm{P}_{\mathrm{k}}(\mathrm{t})=\sum_{n=k}^{\infty} \frac{n !}{k ! n-k !} \mathrm{P}_{0}{ }^{k}\left(1-\mathrm{P}_{\mathrm{o}}\right)^{\mathrm{n}-\mathrm{k}} \mathrm{P}_{\mathrm{n}}(\mathrm{t}) \\
& =\mathrm{P}_{\mathrm{o}}{ }^{k} \frac{(\lambda t)^{k}}{k !} e^{-\lambda P_{o} t}
\end{aligned}
$$

Hence,

$$
\mathrm{P}_{\mathrm{k}}(\mathrm{t})=\frac{\left(\lambda_{o} t\right)^{k}}{k !} e^{-\lambda_{o} t}
$$

where,

$$
\lambda_{\mathrm{o}}=\lambda \mathrm{P}_{\mathrm{o}}
$$

From (7) it is clear that the output number of processed pulses during time $t$ is random variable distributed according to Poisson distribution with parameter $\lambda_{0}$. In the following sections we will study the effect of the time overlapping pulses on the receiver operation in both cases of paralayzable and non- paralayzable receiver type.

\section{EFFECT OF THE TIME OVERLAPPING PULSES ON THE BEHAVIOR OF ESM RECEIVER}

Time overlapping between pulses occurs when a new pulse arrives at the input of an ESM receiver before the ESM receiver completes the processing of the previous pulse. The time overlapping degraded the performance of the ESM receiver since there will be missing pulses at the receiver output and sometimes non correct measurements. This explain why the probability of successfully processed pulse $P_{0}$ is less than 1 . The value of $P_{0}$ is evaluated according to the type of processing as shown in the following subsections. 


\subsection{CASE OF PARALAYABLE RECEIVER}

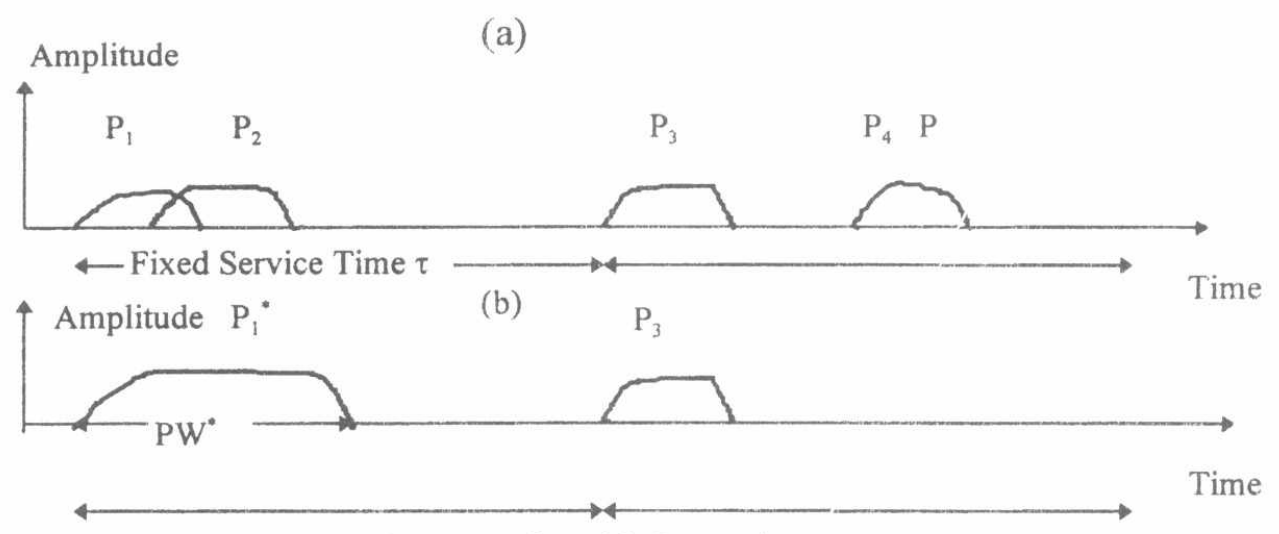

Fig. 4. Paralayzable Processing

(a) Arrival pulse train to the input of an ESM receiver

(b) Output pulse train of the receiver

Fig.4.a and Fig.4.b illustrate the phenomenon of pulse overlapping and its effect on the operation of the paralayzable receiver. The first two pulses P1 and P2 are time overlapped so, they will be processed as a single pulse $\mathrm{P}^{*}$ with new pulse width $\mathrm{PW}^{*}$, after the trailing edge of this pulse the receiver is blocked till the end of fixed service time $\tau$. Although pulse P3 and P4 are not time overlapped after the trailing edge of pulse P3 the receiver is blocked till the end of the fixed service time $\tau$, which result in missing of pulse P4. In this type of receiver the probability of correct processed pulse is the probability that the interarrival time between the successive pulses is greater than the fixed service time $\tau$ of the receiver. So, $P_{0}$ can be calculated according to the following equation:

$$
\mathrm{P}_{\mathrm{o}}=\int_{\tau}^{\infty} \lambda e^{-\lambda t} d t=\mathrm{e}^{-\lambda \tau} \quad \text { (9) (Paralayzable mode) }
$$

A computer program is constructed to simulate the operation of the paralayzable receiver type ,where the ratio of correctly processed pulses is computed as a function of the input arrival rate $\lambda$. The results are compared with the obtained analytic form in equation (9). The results are shown in Fig. 5 where we can see that both the calculated analytic form and the computer simulation results are approximately the same. 
Proceeding of the $\mathbf{1}^{\text {st }}$ ICEENG conference, 24-26 March, 1998.

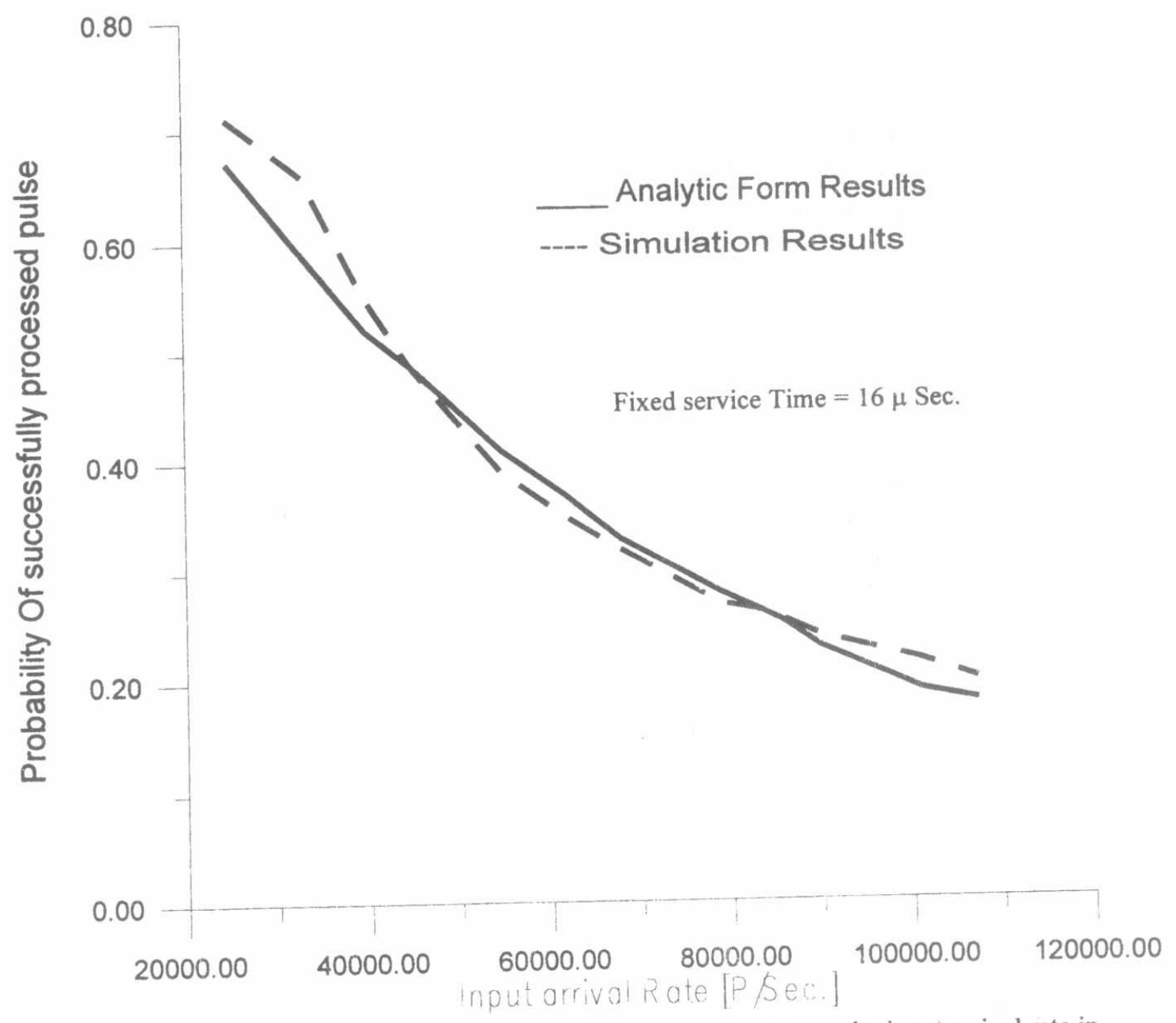

Fig. 5. Probability of successfully processing versus the input arrival rate in paralayzable processing mode

Also, we can see that the ratio of successfully processed pulses is inversely proportional to the input arrival rate of radar pulses at the antenna of an ESM receiver.

\section{5-2 Case Of Non Paralayzable Receiver}

Amplitud

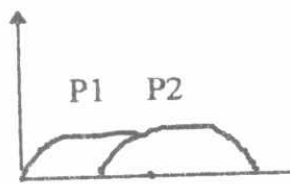

Amplitude

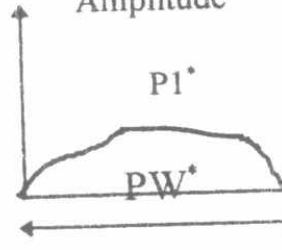

(a)

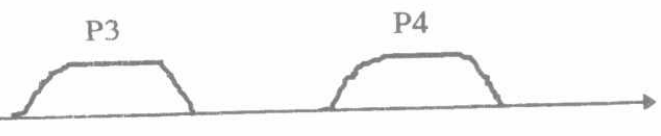

Time

(b)

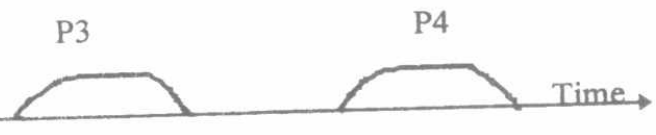

Fig. 6. Non paralayzable processing

(a) Arrival pulse train to the input of an ESM receiver

(b) Output pulse train of the receiver 
Fig.6.a and Fig.6.b illustrate the phenomenon of pulse overlapping and its effect on the operation of the non paralayzable receiver. The first two pulses $\mathrm{P} 1$ and $\mathrm{P}^{\prime} 2$ are overlapped so, they will be processed as a single pulse $\mathrm{P}^{\circ}$ with new pulse width $\mathrm{PW}^{\circ}$, after the trailing edge of this pulse the receiver is ready to process a new pulse. Pulses $\mathrm{P} 3$ and $\mathrm{P} 4$ are riot overlapped so, both of them will be correctly processed in opposite to the previous case of paralayzable receiver where pulse P4 was missed.. The service time or the processing time of the received pulse in a non paralayzable receiver is equal to the pulse width of this pulse. The pulse width of the processed pulse is random variable distributed according to negative exponential distribution with parameter $\mu$ which is the inverse of the average pulse width of all radars expected to exist in the ESM receiver environments. So, in this type the queuing model that describe the operation of the non paralayzable receiver is $(\mathrm{M} / \mathrm{M} / 1)$ model . First $\mathrm{M}$ means that the interarrival time between received pulses at the E.SM receiver input is random variable distributed according to negative exponential distribution with parameter $\lambda$ in our case, the second $M$ means that the service time is random variable distributed according to negative exponential distribution with parameter $\mu$, and the last symbol means that there is one receiver (server) to process the intercepted radar pulses. The non- paralayzable receiver operations can be represented by a finite state machine of two states as shown in Fig. 7 .

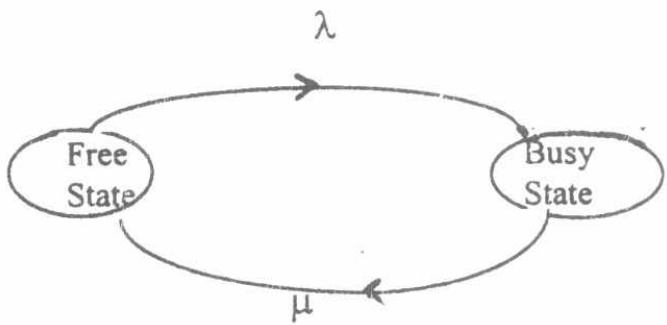

Fig.7. finite state machine representation of non paralayzable receiver mode

It is easy to drive that the ratio of successfully processed pulses is

$$
\mathrm{P}_{0}=\frac{\mu}{\lambda+\mu} \quad \text { (10) (Non paralayzable mode) }
$$

This result is agree with one obtained in [2] . A computer program is constructed to simulate the operation of non- paralayzable receiver The results prove that the output PDVs emerge from the receiver and encoder subsystem according to Poisson distribution with parameter $\lambda_{0}$, and the ratio of successfully processed pulses $P_{0}$ is as given in equation (10). 


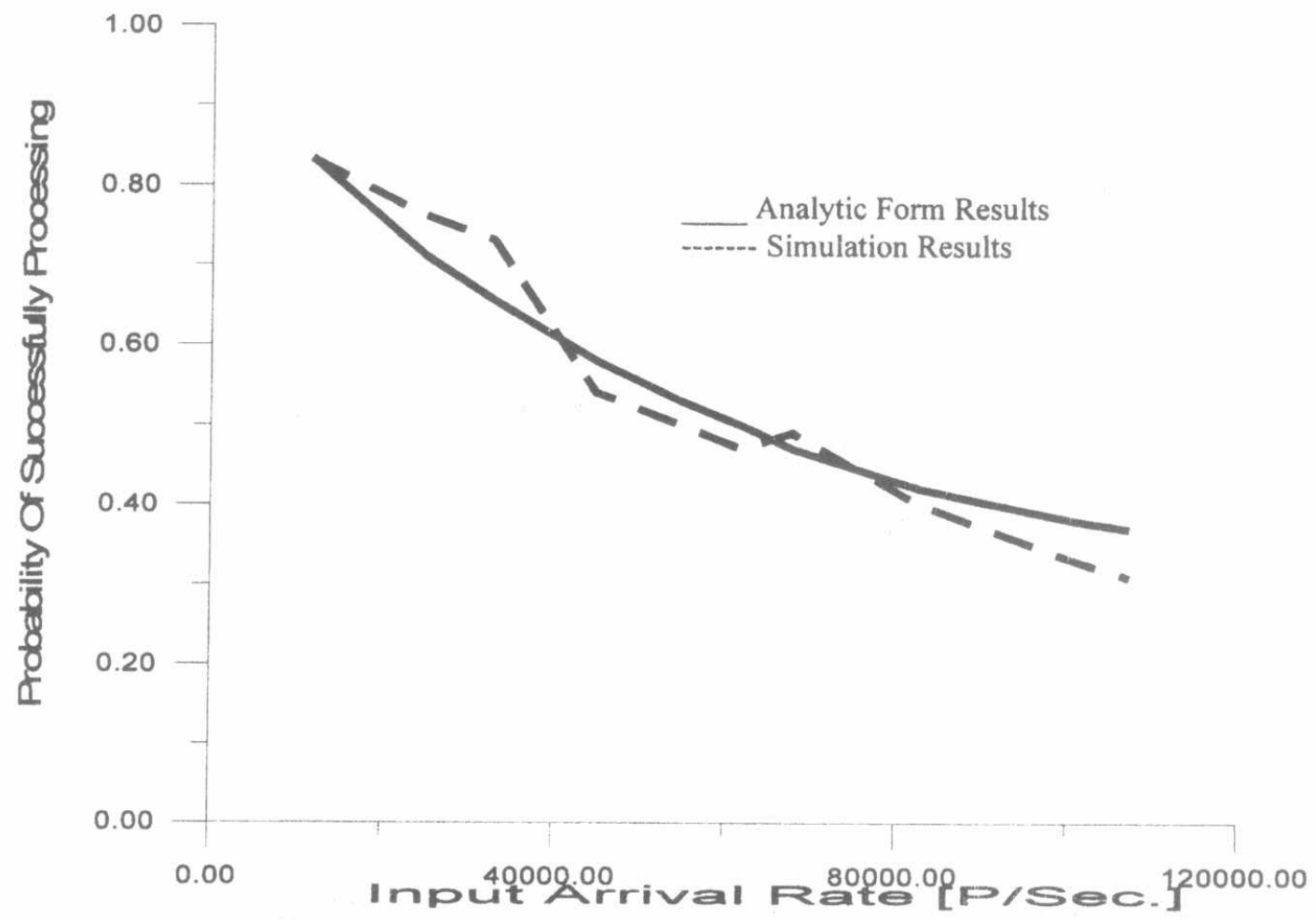

Fig. 8. The Probability of successfully processing of pulses versus the input arrival rate in case of non paralayzable processing

Fig. 8 shows the simulated and the calculated ratio of correctly processed pulses with respect to the input arrival rates.It is clear that the ratio of successfully processed pulses is inversely proportional to the input arrival rate which is the same result in case of paralayzable receiver. It worth to note that in case of many overlapped pulses the non paralayzable receiver operates with the resultant pulse width of the overlapped pulses. The resultant pulse width must be less than certain value which is chosen to be the maximum pulse width expected of all radars in the environment of the ESM receiver. If the resultant pulse width is greater than that value, the receiver consider that the pulse width is equal to that maximum value. This consideration overcome overloading problem by a CW jamming signal.

Fig. 9 shows a comparison between the paralayzable and non paralayzable receivers from the point of view of the ratio of successfully processed pulses versus the input arrival rate. In this work we consider that the fixed service time $\tau$ of paralayzable processing is approximately equal to average pulse width of all radars in the ESM receiver environments $(1 / \mu)$. The figure indicates that the ratio of successfully processed pulses in case of non paralayzable receiver is greater than the same ratio in case of paralayzable receiver, at the same input arrival rate of received pulses. 
Proceeding of the $1^{\text {st }}$ ICEENG conference, 24-26 March, 1998.

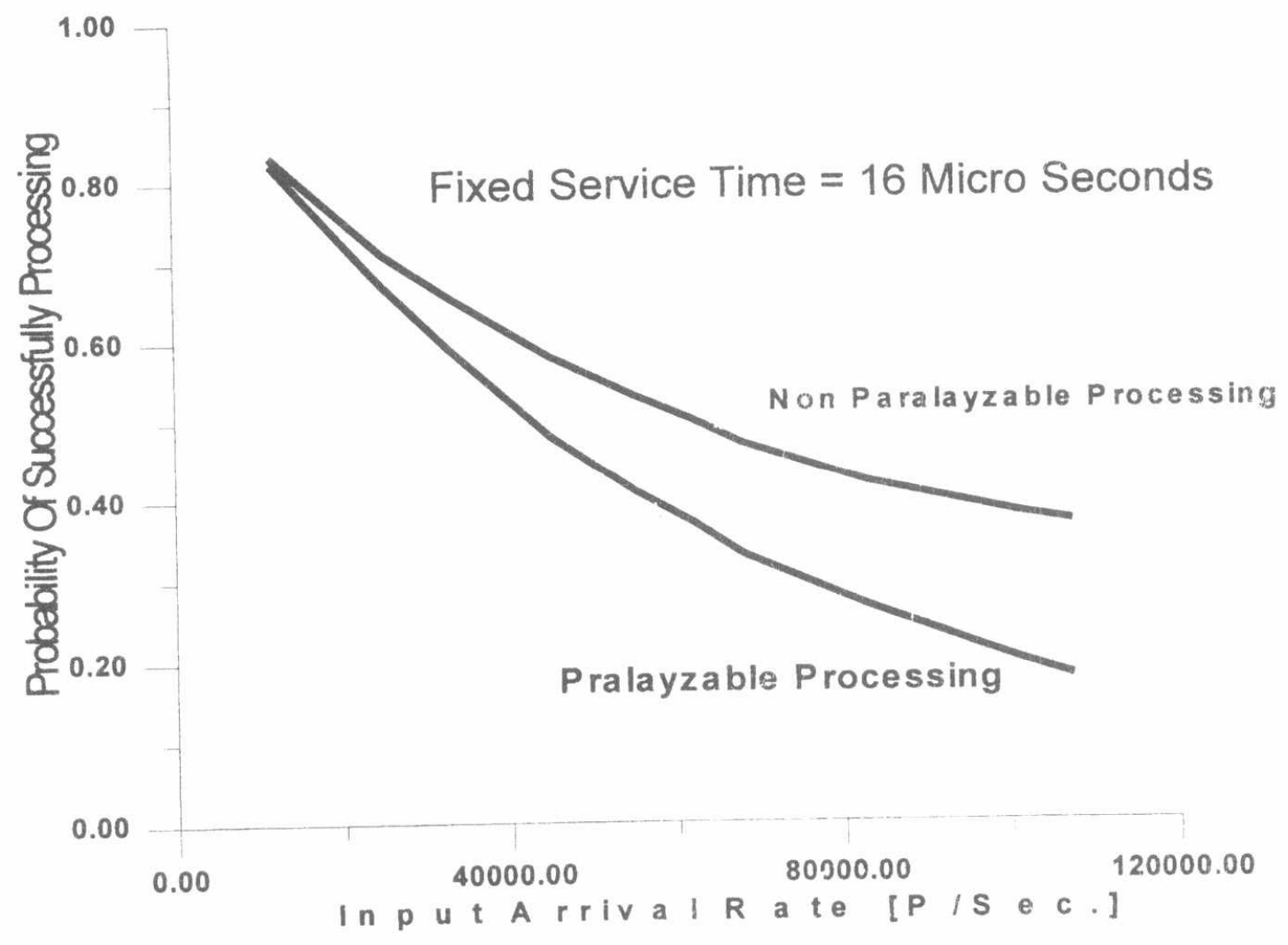

Fig. 9. Comparison of the probability of successfully processing as a function of input arrival rate

\section{Conclusion}

In this paper a mathematical model representing the environments of an ESM receiver is constructed. The environments are assumed to consist of $\mathrm{N}$ - periodic radars with rotating antennas . The resultant arrival process to the ESM receiver is found to be a random process with Poisson distribution. Moreover the interarrival time betwcen the received pulses is a negative exponential distributed random variable with an average arrival rate equal to the sum of the pulse repetition frequencies of the existing radars in the environments. A computer program simulating the environments is constructed. The simulation results supports the obtained analytic forms. The problem of overlapping pulses is considered in both cases of paralayzable and non paralayzable processing. Analytic forms for the ratio of successfully processed pulses, relative to the input pulse train is derived in both cases of processing. The obtained results are compared with the results of the computer simulation. The results show that the non paralayzable processing reduces the number of missing pulses and improve the probability of successfully processing compared with the paralayzable processing.

\section{References}

[1] Bussgang, J. J and Fine, T. L., "Interpulse interval distribution in the environment of N periodic pulse radars," IEEE Trans. On Radio Frequency Interference, vol. RFI-5 , pp 7-10,(1963).

[2] Bunday D., Basic Queuing Theory, Edward Arnold, (1986).

[3] DAVIES , C.L., and HOLLANDS, P. "Automatic processing for ESM." IEE Proc. F, Commun. , Radar \& Signal Process., 129, (3), pp 164-171, (1982) 